PharmacoEconomics \& Outcomes News 882, p8 - 10 Jul 2021

\title{
COVID-19-related decreases in paediatric PCV 13 use does not justify vaccination of older adults
}

The results of a US-based analysis examining COVID-19-related changes in paediatric 13-valent pneumococcal conjugate vaccine (PCV 13) use and its subsequent impact on pneumococcal disease rates do not support PCV13 use in non-immunocompromised adults aged $\geq 65$ years.

The cost-effectiveness analysis published in Vaccine used a Markov model to estimate paediatric disease resulting from decreased PCV13 use in children aged $<5$ years, with absolute decreases ranging from $10 \%-50 \%$ over $1-2$ years' duration examined. The model assumed no catch-up vaccination and that decreased vaccination led to proportionate increases in pneumococcal disease in children and older adults. The output of this model was then used to populate a second Markov model that estimated the cost effectiveness of older adult pneumococcal vaccination strategies accommodating potential epidemiologic changes from decreased paediatric PCV 13 vaccination.

One year of $10 \%-50 \%$ absolute decreases in PCV 13 use in the under 5-year-olds increased pneumococcal disease by around $4 \%-19 \%$ in $\geq 65$ year olds; after 2 years of decreased PCV 13 use, disease rates increased in older patients by $8 \%-38 \%$. However, at a cost-effectiveness threshold of \$US200 000 per quality-adjusted life-year (QALY) gained, PCV 13 use in all $\geq 65$ year-olds would only be favoured when there was a $>53 \%$ increase in pneumococcal disease, this corresponded to absolute decreases in paediatric PCV 13 vaccination of $>50 \%$ over a 2-year period. An alternative vaccination strategy (PCV 13 of immunocompromised $\geq 65$ year-olds only) was favoured at a the $\$ 200000$ per QALY gained threshold when the relative increase in senior pneumococcal disease rates was $>10 \%$, corresponding to decreases in paediatric PCV 13 use of $>30 \%$ over 1 year and $>20 \%$ over 2 years.

"Decreases in pediatric PCV 13 use, which could increase pneumococcal disease incidence in both children and seniors, would need to be substantial and prolonged to influence the cost-effectiveness of the current pneumococcal vaccination recommendations for adults 65 years old", conclude the authors.

Smith KJ, et al. Should older adult pneumococcal vaccination recommendations change due to decreased vaccination in children during the pandemic? A cost-effectiveness analysis. Vaccine : 12 Jun 2021. Available from: URL: http://doi.org/10.1016/j.vaccine.2021.06.037 